Check for updates

Cite this: Chem. Commun., 2017, 53,9498

Received 6th July 2017,

Accepted 31st July 2017

DOI: $10.1039 / c 7 c c 05215 d$

rsc.li/chemcomm

\section{Evidence and isolation of tetrahedral intermediates formed upon the addition of lithium carbenoids to Weinreb amides and $N$-acylpyrroles $\dagger$}

\author{
Laura Castoldi, Wolfgang Holzer, Thierry Langer and Vittorio Pace (D) *
}

\begin{abstract}
The tetrahedral intermediates generated upon the addition of halolithium carbenoids ( $\mathrm{LiCH}_{2} \mathrm{X}$ and $\mathrm{LiCHXY}$ ) to Weinreb amides have been intercepted and fully characterized as O-TMS heminals. The commercially available $N$-trimethylsilyl imidazole is the ideal trapping agent whose employment, combined with a straightforward neutral Alox chromatographic purification, enables the isolation of such labile species. The procedure could be advantageously extended also for obtaining $\mathrm{O}$-TMS heminals from $\mathrm{N}$-acylpyrroles. These intermediates manifest interesting reactivity including as precursors of more complex carbenoids.
\end{abstract}

The addition reactions of nucleophiles to the amide carbonyl constitute extremely important chemical transformations governing key cascades in biological systems and fundamental synthetic operations. ${ }^{1}$ Mechanistically, a given nucleophile adds to a $\mathrm{C}=\mathrm{O}$ functionality affording a tetrahedral intermediate which, depending on the reaction conditions, could restore the carbonyl group (via the exclusion of a suitable leaving group, i.e. additionelimination pathway). Conversely - whenever the leaving group could not be eliminated - the overall process upon simple acidolysis affords an hydroxyl-type final product. ${ }^{2}$ For a long time, the tamed reactivity of amides towards carbon nucleophiles compared to other carboxylic acid derivatives has been rewarded as a limitation, thus considering them reluctant species in analogous processes (Scheme 1). ${ }^{3}$ Nowadays, the opportune activation of the amide bond with electrophilic reagents represents an excellent and robust tool for enabling the chemoselective addition of organometallic reagents en route to the synthesis of ketones, alcohols, or amines as documented in illuminating recent works by Charette, ${ }^{4}$ Huang ${ }^{5}$ and Movassaghi. ${ }^{6}$ Undoubtedly, the introduction by Nahm and Weinreb of $N$-methoxy- $N$-methyl amides in $1981^{7}$ represented a breakthrough in the field and currently they are routinely employed for accessing carbonyls with high chemocontrol. ${ }^{8}$ The complexation of the metal of the putative tetrahedral intermediate

University of Vienna - Department of Pharmaceutical Chemistry, Althanstrasse, 14, A-1090, Vienna, Austria. E-mail: vittorio.pace@univie.ac.at

$\dagger$ Electronic supplementary information (ESI) available. See DOI: 10.1039/c7cc05215d
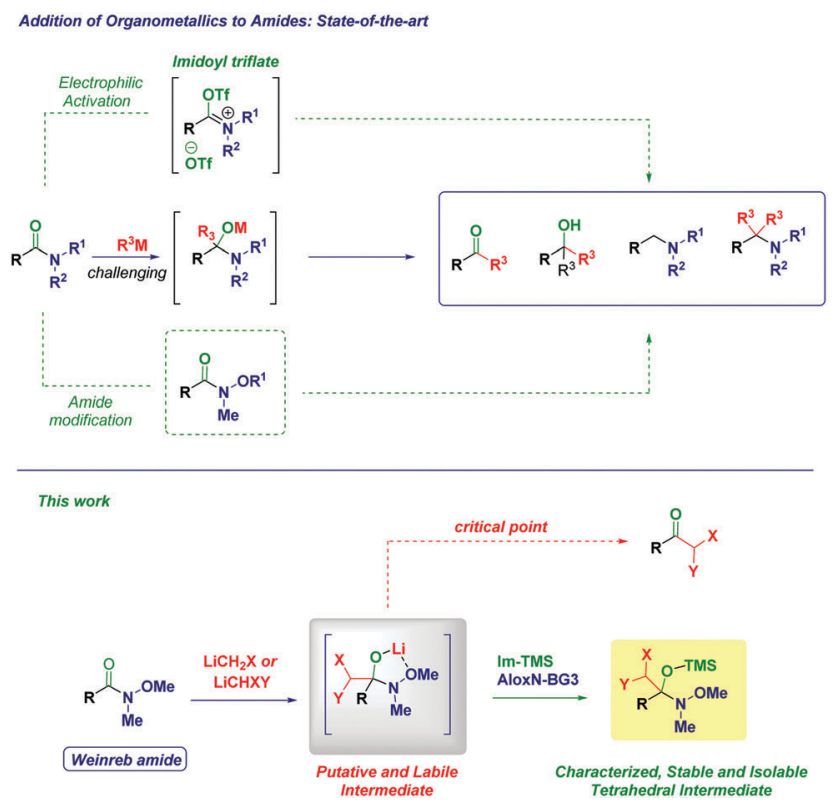

Scheme 1 General context of the presented work.

to form a five-membered cycle is the critical factor justifying the effectiveness in hampering common undesired drawbacks such as overaddition phenomena or low reactivity. Further advancements on $N$-alkoxy amides pointed out the feasibility of the addition of two different organometallics as showcased recently in elegant works by Chida-Sato, ${ }^{9}$ Sarpong, ${ }^{10}$ Helmchen, ${ }^{11}$ Feringa, ${ }^{12}$ Schwartz ${ }^{13}$ and Vincent-Kouklovsky ${ }^{14}$ thus, disclosing new concepts for carbonyl and amine synthesis. Additionally, the recent development of impressive catalytic tactics for enabling the reactivity of amide carbonyls with organometallic reagents by the group of Garg $^{15}$ and Szostak ${ }^{16}$ opened new avenues for the use of amide as feedstocks in analogous processes.

Recently, we recognized the excellent performance of Weinreb amides as privileged acylating agents for chemoselective homologations with $\alpha$-substituted methyllithium reagents $\left(\mathrm{LiCH}_{2} \mathrm{X}\right){ }^{17}$ Compared to more classical substrates for similar reactions 
(e.g. esters), Weinreb amides showed an impressive superiority, which we attributed to the chelating effect typical of these species. However, the inherent transient nature of the tetrahedral intermediate $^{18}$ formed through the addition of a strongly nucleophilic organometallic reagent implies severe difficulties in isolating and identifying via spectroscopic methods these species and, ultimately to fully uncover their synthetic potential. ${ }^{19}$ With the aim to support these hypotheses, we document herein the isolation and the structural elucidation of $O$-trimethylsilyl protected hemiaminals obtained via the addition of lithium halocarbenoids $\left(\mathrm{LiCH}_{2} \mathrm{X} \text { and } \mathrm{LiCHXY}\right)^{20}$ to variously functionalized Weinreb amides and analogous $N$-acylpyrroles. ${ }^{21}$

At the outset of our investigations, we easily recognized the importance of finding suitable reaction conditions and work-up operations enabling not only the safe capture but, more importantly, also preserving the chemical integrity of the labile tetrahedral intermediate. The ideal trapping agent should present a remarkable oxophilicity and contemporaneously provide the sufficient stability to avoid the undesired elimination of the $N$-methyl- $N$-methoxy fragment, which would have afforded the corresponding ketone. Cognizant of these critical requirements, Weinreb amide 1 was selected as the model substrate and reacted with $\mathrm{LiCH}_{2} \mathrm{Cl}$ (Table 1) under our usual Barbier-type conditions. ${ }^{17 c, 22}$ Neither through basic aqueous or methanolic work-up could the free hemiaminal be isolated. TMSCl in the presence of pyridine (entry 1) served as an effective trapping agent (3) as deduced from the diagnostic hemiaminal (carbon) ${ }^{13} \mathrm{C}(96.0 \mathrm{ppm})$ and (nitrogen) ${ }^{15} \mathrm{~N}-\mathrm{NMR}(-207.3 \mathrm{ppm})$ resonances. The employment of imidazole as the base was particularly convenient since the conversion could be increased up to 90\% (entry 2). Pleasingly, the commercially available $N$-TMSimidazole (Im-TMS) allowed to maximize the conversion up to $95 \%$ (entry 3), whereas the more bulky TBDMS and TPS

Table 1 Reaction and isolation procedure optimization ${ }^{a}$

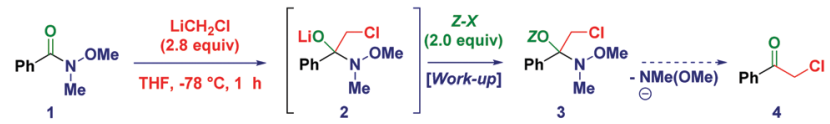

$$
\begin{aligned}
& Z-X=\text { Trapping agent }
\end{aligned}
$$

\begin{tabular}{|c|c|c|c|c|}
\hline Entry $^{a}$ & $\begin{array}{l}\text { Trapping agent } \\
\text { (2.0 equiv.) }\end{array}$ & $\begin{array}{l}\text { Conversion/ } \\
\text { ratio } 3: \mathbf{4}^{b}(\%)\end{array}$ & $\begin{array}{l}\text { Chromatographic } \\
\text { system }\end{array}$ & $\begin{array}{l}\text { Yield of } \\
3^{c, d}(\%)\end{array}$ \\
\hline 1 & TMSCl-Py & $87 / 5: 1$ & AloxN-BG3 & 71 \\
\hline 2 & TMSCl-Im & $90 / 20: 1$ & AloxN-BG3 & 70 \\
\hline 3 & Im-TMS & $95 / 25: 1$ & AloxN-BG3 & 86 \\
\hline 4 & Im-TBDMS & $86 / 0: 1$ & - & - \\
\hline 5 & Im-TPS & $89 / 0: 1$ & - & - \\
\hline 6 & Im-TMS & $95 / 25: 1$ & $\mathrm{SiO}_{2}$ & 0 \\
\hline 7 & Im-TMS & $95 / 25: 1$ & $\mathrm{SiO}_{2}$-TEA $(10 \%)$ & 6 \\
\hline 8 & Im-TMS & $95 / 25: 1$ & $\mathrm{SiO}_{2}$-TMSCl (5\%) & 12 \\
\hline 9 & Im-TMS & $95 / 25: 1$ & AloxN-BG3 & 91 \\
\hline 10 & Im-TMS & $95 / 25: 1$ & AloxN-BG4 & 78 \\
\hline 11 & Im-TMS & $95 / 25: 1$ & AloxN-BG2 & 70 \\
\hline
\end{tabular}

${ }^{a} \mathrm{LiCH}_{2} \mathrm{Cl}$ was generated from $\mathrm{ICH}_{2} \mathrm{Cl}$ (3.0 equiv.) and $\mathrm{MeLi}-\mathrm{LiBr}$ (2.8 equiv.) in THF at $-78{ }^{\circ} \mathrm{C} .{ }^{b}{ }^{1} \mathrm{H}$-NMR calculated conversion. ${ }^{c}$ Otherwise stated, yields refer to isolated and purified compounds according to conditions in entry 9 (AloxN-BG3). ${ }^{d}$ For the preparation of AloxN-BG3, see the ESI. reagents afforded exclusively the corresponding $\alpha$-chloroketone (entries 4-5), presumably due to steric factors affecting negatively the stability of the intermediate. The work-up and purification technique followed for getting analytically pure 3 deserve particular mention. After basic aqueous treatment $\left(\mathrm{NaHCO}_{3} 5 \%\right)$ and extraction of the organic phase with $\mathrm{Et}_{2} \mathrm{O}$, we noticed a substantial transformation of the product into ketone $\mathbf{4}$ during recording of NMR spectra in $\mathrm{CDCl}_{3}$, presumably because of the acidity of this deuterated solvent. Analogously, attempts to purify the crude mixture through silica gel were unsuccessful, even upon prior neutralizing treatment with TEA (10\% or neat) or TMSCl (entries 6-8). Excellent purification was achieved by using Brockmann 3 grade neutral alumina (AloxN-BG3), which allowed us to finally isolate 3 in 91\% yield (entry 9). Different deactivation grades of Alox were less effective (entries 10-11), while different alumina type phases (acidic or basic) were not suitable for the purpose (not shown).

With the optimized conditions in hand, we then applied the methodology to different Weinreb amides (Scheme 2). The corresponding tetrahedral intermediates could be easily isolated and characterized in high to excellent chemical yields. Accordingly, aromatic (3-11) and heteroaromatic (12-13) Weinreb amides provided the $O$-TMS protected hemiaminals regardless of the electronic behavior of the substituents across the ring. Notably, the presence of an exchangeable iodine atom on the aromatic ring did not have a detrimental effect (10), thus highlighting an excellent chemoselectivity during the carbenoid generation event starting from $\mathrm{ICH}_{2} \mathrm{Cl}$. Switching to aliphatic analogues (14-15), no alteration was observed during the trapping-isolation sequence even in the presence of a $\alpha$-heteroatom (15). Tetrahedral intermediates
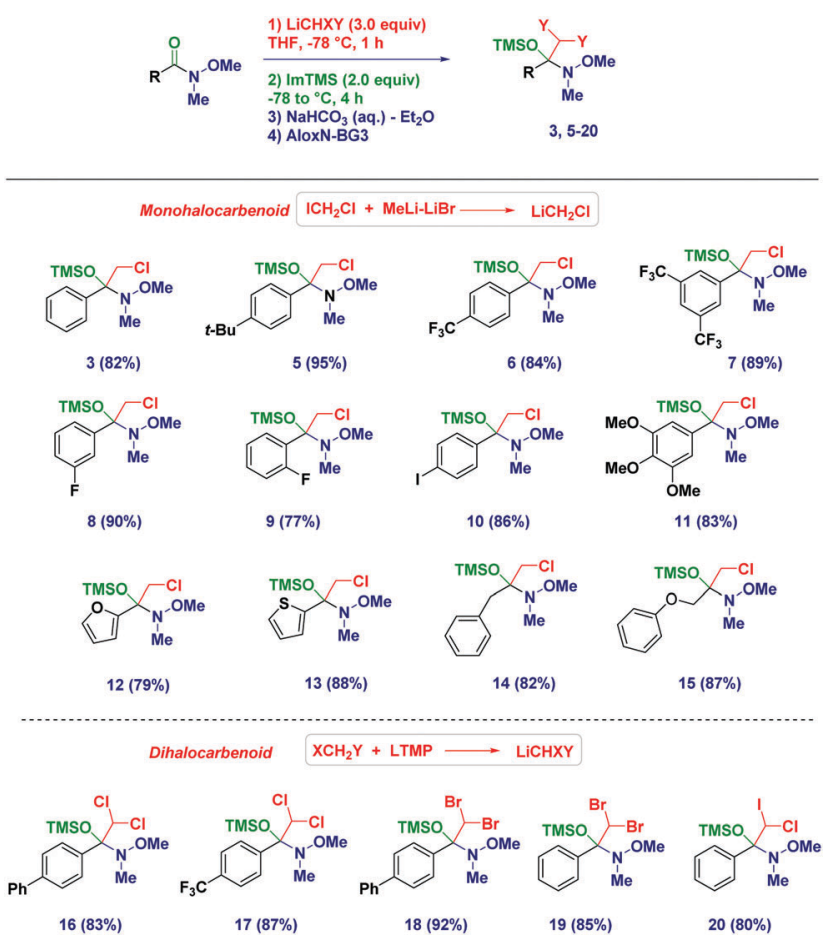

Scheme 2 Trapping-isolation sequence of O-TMS protected aminals from Weinreb amides with monohalo- and dihalomethyllithium carbenoids. 


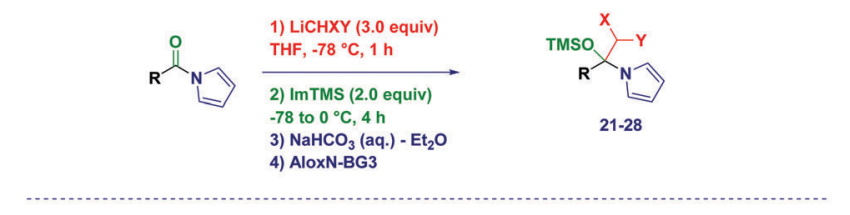

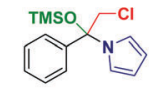

$21(80 \%)$

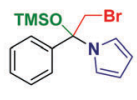

$22(77 \%)$
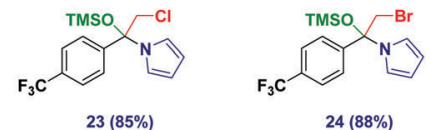

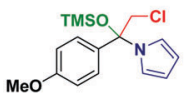

25 (76\%)

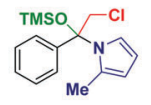

$26(60 \%)$

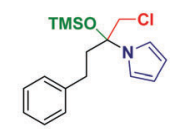

27 (79\%)

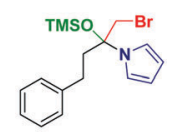

$28(82 \%)$
Scheme 3 O-TMS protected hemiaminals from $\mathrm{N}$-acylpyrroles and carbenoids.

generated upon the addition of (mixed) dihalocarbenoids ${ }^{23}$ could be easily accessed (16-20) and could serve as excellent placeholders for forming more complex, synthetically versatile carbenoids (vide infra). Remarkably, $\mathrm{C}_{6} \mathrm{D}_{6}$ solutions of the intermediates showed an excellent stability up to 6 months (kept at $-20{ }^{\circ} \mathrm{C}$ ), thus further showcasing the convenience of this solvent for NMR analyses.

The protocol was also amenable for forming the tetrahedral intermediates - under analogous reaction conditions - starting from $N$-acylpyrroles (Scheme 3). Again the aromatic (21-26) vs. aliphatic (27-28) nature of the materials did not influence the positive outcome of the process, which significantly also proved to be applicable to intermediates that formed upon the addition of $\mathrm{LiCH}_{2} \mathrm{Br}^{24}$ However, the increase of sterical demand on the pyrrole core slightly decreases the isolated yield of the protected hemiaminal (26).

Previous reports from Evans ${ }^{25}$ and Brandänge ${ }^{26}$ entailed the formation and isolation of hemiaminals from $\mathrm{N}$-acylpyrroles and $\mathrm{RLi}$ or $\mathrm{RMgX}$ in the native form (i.e. presenting a free $\mathrm{OH}$ functionality). ${ }^{27}$ However, in the cases discussed herein, the plausible intramolecular formation of a halogen bond ${ }^{28}$ between the putative $\mathrm{OH}$ and the halogen would trigger the elimination and, as such, it renders it impossible to obtain analogous hemiaminals (Scheme 4).

The reactivity of the synthesized tetrahedral intermediates was then tested with the aim of investigating the synthetic utility of these species (Scheme 5). The iodo-containing intermediate 10 resulted in an excellent substrate for the Feringa/Fañanas-Mastral

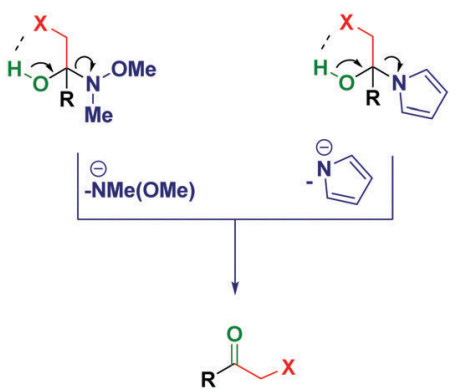

Scheme 4 Plausible halogen-bond formation on putative hemiaminals.

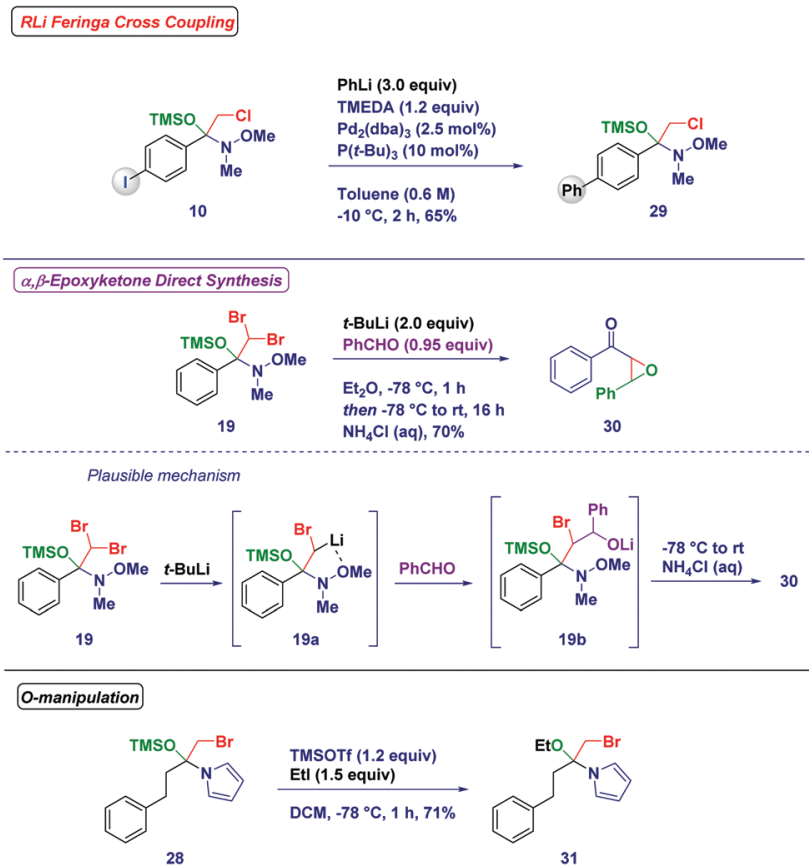

Scheme 5 Derivatization of O-TMS protected hemiaminals.

Pd-catalyzed coupling of an organolithium. ${ }^{29}$ Significantly, under the reaction conditions the tetrahedral intermediate could be completely preserved from degradation (29). An $O$-TMS protected hemiaminal (19) featuring two bromine atoms was amenable to subsequent $\mathrm{Li} / \mathrm{Br}$ exchange to generate a new, more complex carbenoid (19a) whose stability was increased by the chelating effect of the $\mathrm{N}$-methoxy group. The attack of this carbenoid to benzaldehyde generated an $O$-lithiated bromohydrin (19b), which upon spontaneous ring-closure and acidic treatment resulted in the formation of the highly valuable and challenging $\alpha, \beta$ epoxyketone $30 .{ }^{30}$ Moreover, Lewis acid treatment (TMSOTf) of the $N$-acylpyrrole derivative hemiaminal 28 followed by trapping with ethyl iodide afforded the $O$-ethyl product 31 .

In summary, we have disclosed an effective strategy for obtaining O-trimethylsilyl protected hemiaminals formed through the addition of lithium monohalo- and dihalo-carbenoids to Weinreb amides. The proper combination of Im-TMS (as the trapping agent) and Brockmann grade 3 neutral alumina (as the stationary phase for chromatography) was found to be - together with recording NMR analyses in benzene- $d_{6}$ - the critical factor for enabling the isolation of such labile tetrahedral intermediates. The method is also amenable for forming analogous protected hemiaminals from $N$-acylpyrroles. A concise examination of the manipulation these species may undergo is also discussed.

The authors gratefully acknowledge the generous support and a doctoral Uni:docs grant to L.C. from University of Vienna.

\section{Notes and references}

1 (a) V. R. Pattabiraman and J. W. Bode, Nature, 2011, 480, 471; (b) The Amide Linkage. Selected Structural Aspects in Chemistry, Biochemistry and Materials Science, ed. A. Greenberg, C. M. Breneman and J. F. Liebman, John Wiley \& Sons Inc., New York, 2000. 
2 M. B. Smith and J. March, March's Advanced Organic Chemistry: Reactions, Mechanisms, and Structure, Wiley-VCH, Weinheim, 5th edn, 2001.

3 Reviews: (a) V. Pace, W. Holzer and B. Olofsson, Adv. Synth. Catal., 2014, 356, 3697; (b) V. Pace and W. Holzer, Aust. J. Chem., 2013, 66, 507; (c) D. Seebach, Angew. Chem., Int. Ed., 2011, 50, 96.

4 (a) A. B. Charette and M. Grenon, Can. J. Chem., 2001, 79, 1694; (b) W. S. Bechara, G. Pelletier and A. B. Charette, Nat. Chem., 2012, $4,228$.

5 (a) K.-J. Xiao, J.-M. Luo, X.-E. Xia, Y. Wang and P.-Q. Huang, Chem. - Eur. J., 2013, 19, 13075; (b) K.-J. Xiao, A.-E. Wang, Y.-H. Huang and P.-Q. Huang, Asian J. Org. Chem., 2012, 1, 130; (c) K.-J. Xiao, A.-E. Wang and P.-Q. Huang, Angew. Chem., Int. Ed., 2012, 51, 8314; (d) K.-J. Xiao, Y. Wang, K.-Y. Ye and P.-Q. Huang, Chem. - Eur. J., 2010, 16, 12792; (e) K.-J. Xiao, J.-M. Luo, K.-Y. Ye, Y. Wang and P.-Q. Huang, Angew. Chem., Int. Ed., 2010, 49, 3037.

6 J. W. Medley and M. Movassaghi, Angew. Chem., Int. Ed., 2012, 51, 4572.

7 S. Nahm and S. M. Weinreb, Tetrahedron Lett., 1981, 22, 3815.

8 Review: (a) S. Balasubramaniam and I. S. Aidhen, Synthesis, 2008, 3707; For a concise overview on $N$-alkoxyamide, see: $(b)$ T. Sato and N. Chida, Org. Biomol. Chem., 2014, 12, 3147; For the development of new acylating reagents ( $N$-acylazetidines), see: $(c) \mathrm{C}$. Liu, M. Achtenhagen and M. Szostak, Org. Lett., 2016, 18, 2375.

9 (a) K. Shirokane, Y. Kurosaki, T. Sato and N. Chida, Angew. Chem., Int. Ed., 2010, 49, 6369; (b) Y. Kurosaki, K. Shirokane, T. Oishi, T. Sato and N. Chida, Org. Lett., 2012, 14, 2098; (c) Y. Oda, T. Sato and N. Chida, Org. Lett., 2012, 14, 950; (d) Y. Yanagita, H. Nakamura, K. Shirokane, Y. Kurosaki, T. Sato and N. Chida, Chem. - Eur. J., 2013, 19, 678; (e) K. Shirokane, T. Wada, M. Yoritate, R. Minamikawa, N. Takayama, T. Sato and N. Chida, Angew. Chem., Int. Ed., 2014, 53, 512; $(f)$ M. Yoritate, T. Meguro, N. Matsuo, K. Shirokane, T. Sato and N. Chida, Chem. - Eur. J., 2014, 20, 8210; (g) M. Nakajima, Y. Oda, T. Wada, R. Minamikawa, K. Shirokane, T. Sato and N. Chida, Chem. - Eur. J., 2014, 20, 17565.

10 S. T. Heller, J. N. Newton, T. Fu and R. Sarpong, Angew. Chem., Int. Ed., 2015, 54, 9839.

11 M. Jäkel, J. Qu, T. Schnitzer and G. Helmchen, Chem. - Eur. J., 2013, 19, 16746 .

12 M. Giannerini, C. Vila, V. Hornillos and B. L. Feringa, Chem. Commun., 2016, 52, 1206.

13 J. Nugent and B. D. Schwartz, Org. Lett., 2016, 18, 3834.

14 G. Vincent, R. Guillot and C. Kouklovsky, Angew. Chem., Int. Ed., 2011, 50, 1350.

15 (a) J. E. Dander and N. K. Garg, ACS Catal., 2017, 7, 1413; For a breakthrough in the field, see: $(b)$ L. Hie, N. F. Fine Nathel, T. K. Shah, E. L. Baker, X. Hong, Y.-F. Yang, P. Liu, K. N. Houk and N. K. Garg, Nature, 2015, 524, 79.

16 (a) G. Meng and M. Szostak, Org. Lett., 2015, 17, 4364; (b) G. Meng, S. Shi and M. Szostak, ACS Catal., 2016, 6, 7335.
17 (a) V. Pace, W. Holzer, G. Verniest, A. R. Alcántara and N. De Kimpe, Adv. Synth. Catal., 2013, 355, 919; (b) V. Pace, I. Murgia, S. Westermayer, T. Langer and W. Holzer, Chem. Commun., 2016, 52, 7584; (c) V. Pace, L. Castoldi and W. Holzer, J. Org. Chem., 2013, 78, 7764; (d) A. D. Mamuye, L. Castoldi, U. Azzena, W. Holzer and V. Pace, Org. Biomol. Chem., 2015, 13, 1969; For accounts, see: $(e)$ V. Pace, W. Holzer and N. De Kimpe, Chem. Rec., 2016, 16, 2061; $(f)$ V. Pace, L. Castoldi, S. Monticelli, M. Rui and S. Collina, Synlett, 2017, 879.

18 Review: M. Adler, S. Adler and G. Boche, J. Phys. Org. Chem., 2005, 18, 193.

19 The addition of the Ruppert-Prakash reagent $\left(\mathrm{TMSCF}_{3}\right)$ to Weinreb amides gives trifluoromethyl analogue intermediates: D. M. Rudzinski, C. B. Kelly and N. E. Leadbeater, Chem. Commun., 2012, 48, 9610.

20 Reviews: (a) V. Capriati, Contemporary Carbene Chemistry, John Wiley \& Sons, Inc, 2013, p. 325; (b) V. Capriati and S. Florio, Chem. - Eur. J., 2010, 16, 4152; (c) V. H. Gessner, Chem. Commun., 2016, 52, 12011; (d) S. Molitor and V. H. Gessner, Synlett, 2015, 861; For recent contributions, see: (e) S. Molitor and V. H. Gessner, Angew. Chem., 2016, 128, 7843; $(f)$ S. Molitor, K.-S. Feichtner, C. Kupper and V. H. Gessner, Chem. - Eur. J., 2014, 20, 10752; $(g)$ S. Molitor, J. Becker and V. H. Gessner, J. Am. Chem. Soc., 2014, 136, 15517; (h) V. Pace, L. Castoldi, E. Mazzeo, M. Rui, T. Langer and W. Holzer, Angew. Chem., Int. Ed., 2017, DOI: 10.1002/anie.201706236.

21 Review: A. M. Goldys and C. S. P. McErlean, Eur. J. Org. Chem., 2012, 1877.

22 (a) V. Pace, Aust. J. Chem., 2014, 67, 311; For an interesting report on the use of $\mathrm{LiCH}_{2} \mathrm{X}$ under non-Barbier type conditions via flow chemistry, see: (b) L. Degennaro, F. Fanelli, A. Giovine and R. Luisi, Adv. Synth. Catal., 2015, 357, 21.

23 V. Pace, L. Castoldi, A. D. Mamuye and W. Holzer, Synthesis, 2014, 2897.

24 V. Pace, A. Pelosi, D. Antermite, O. Rosati, M. Curini and W. Holzer, Chem. Commun., 2016, 52, 2639.

25 D. A. Evans, G. Borg and K. A. Scheidt, Angew. Chem., Int. Ed., 2002, 41, 3188.

26 S. Brandänge, E. Holmgren, H. Leijonmarck and B. Rodriguez, Acta Chem. Scand., 1995, 49, 922.

27 For additional rare cases, see: (a) M. Szostak, L. Yao and J. Aubé, J. Am. Chem. Soc., 2010, 132, 2078; (b) M. Szostak and J. Aube, Org. Biomol. Chem., 2011, 9, 27; (c) M. Szostak and J. Aubé, Chem. Rev., 2013, 113, 5701; (d) A. J. Kirby, I. V. Komarov and N. Feeder, J. Am. Chem. Soc., 1998, 120, 7101; (e) J. Halli, K. Hofman, T. Beisel and G. Manolikakes, Eur. J. Org. Chem., 2015, 4624.

28 G. Cavallo, P. Metrangolo, R. Milani, T. Pilati, A. Priimagi, G. Resnati and G. Terraneo, Chem. Rev., 2016, 116, 2478.

29 (a) M. Giannerini, M. Fañanás-Mastral and B. L. Feringa, Nat. Chem., 2013, 5, 667For a highlight, see: (b) V. Pace and R. Luisi, ChemCatChem, 2014, 6, 1516.

30 (a) Q. Ke, B. Zhang, B. Hu, Y. Jin and G. Lu, Chem. Commun., 2015, 51, 1012; (b) W. Liu, Y. Li, K. Liu and Z. Li, J. Am. Chem. Soc., 2011, 133, 10756 . 